\title{
Persistent Antiphospholipid Antibodies, Mast Cell Activation Syndrome, Postural Orthostatic Tachycardia Syndrome and Post-COVID Syndrome: 1 Year On
}

\author{
Jill R Schofield \\ University of Colorado School of Medicine, Aurora, Colorado, USA
}

Received: 07/02/2021

Accepted: 08/02/2021

Published: $22 / 03 / 2021$

How to cite this article: Schofield JR. Persistent antiphospholipid antibodies, mast cell activation syndrome, postural orthostatic tachycardia syndrome and post COVID syndrome: 1 year on. EJCRIM 2021;8: doi:10.12890/2021_002378.

Conflicts of Interests: The Authors declare that there are no competing interests.

This article is licensed under a Commons Attribution Non-Commercial 4.0 License

\section{ABSTRACT}

This is the first case report of a patient with post-COVID-19 postural orthostatic tachycardia syndrome (POTS) with multiple persistent antiphospholipid antibody (aPL)-positivity more than a year after illness onset who also meets Global Consensus-2 criteria for mast cell activation syndrome (MCAS), suggesting pathological activation of the acquired and innate immune systems by SARS-CoV-2. While the patient continues to meet criteria for POTS 1 year on, her functional ability has improved significantly with therapy directed at MCAS, POTS and aPL-positivity.

\section{LEARNING POINTS}

- A subset of long-haul COVID-19 patients have postural orthostatic tachycardia syndrome(POTS), which can be diagnosed by a 10-minute in-office stand test.

- Antiphospholipid antibodies may be associated with POTS in patients with long-haul COVID-19 and have important clinical implications.

- Mast cell activation syndrome (MCAS) may be associated with long-haul COVID-19 (with or without POTS) and can often be easily treated, including with over-the-counter medications, supplements and dietary changes.

\section{KEYWORDS}

Long-haul COVID-19, antiphospholipid antibodies, postural orthostatic tachycardia syndrome, mast cell activation syndrome, post-COVID syndrome

\section{BACKGROUND}

A subset of patients with COVID-19 experience symptoms lasting >12 weeks ${ }^{[1]}$. In May 2020, affected patients began to connect on social media forums and labelled themselves as COVID 'long-haulers'. These groups suspected that some long-haulers may have mast cell activation syndrome (MCAS) and/or postural orthostatic tachycardia syndrome (POTS), which associations have subsequently been reported in the medical literature ${ }^{[2-5]}$ and the media.

The precise pathogenesis of post-COVID-19 syndromes is unknown. There is not usually ongoing viral infection ${ }^{[2]}$ but rather a suspected dysregulated innate and/or acquired immune response triggered by the virus. We report a patient with post-COVID-19 POTS who has objective evidence for both with MCAS and persistent aPL-positivity. She has improved significantly with directed therapy. 


\section{CASE DESCRIPTION}

A 50-year-old business executive was healthy, except for underlying issues suggesting mild MCAS. These included sensitivity to perfumes/ cigarette smoke beginning as a toddler, eczema and environmental allergies beginning in elementary school, diffuse urticaria and episodic migraine beginning in high school, and gastrointestinal reflux disease beginning in adulthood.

In February 2020, she and two colleagues became ill while working in Europe. Her co-workers were diagnosed with COVID-19 upon returning to the USA. She was not PCR-tested until April (negative), but SARS-CoV-2 IgG was positive in June. Her initial symptoms included subjective fever, fatigue, nausea and diarrhoea, but she improved the following day. One week later, she again felt unwell with similar symptoms that were short-lived. On March 24, more severe symptoms began, including low-grade fever, nausea, presyncope, dyspnoea, chest tightness, myalgias, constant thirst, pruritus, hives and temperature dysregulation. After 2 weeks, she gradually improved, but despite rowing 90 minutes daily prior to illness onset, even 10 minutes of exercise caused her symptoms to flare up. A patient forum led her to suspect possible MCAS. She began a low histamine diet and antihistamines which helped. However, symptoms flared again 2 weeks later. With each flare, she became bedbound for a few days, then would gradually improve. After 2 weeks, she recovered to $60 \%$ of normal function and was able to work full-time from home, but had to limit her upright time and struggled to exercise.

When symptoms and flares continued, in July 2020 she underwent cardiopulmonary testing and basic laboratory studies which were normal (Table 1) except for an elevated albumin suggesting hypovolaemia. Hypovolaemia is present in most patients with dysautonomia and hyperalbuminaemia is an important clue suggesting this possibility. No diagnoses were made.

\begin{tabular}{|c|c|c|c|}
\hline \multicolumn{4}{|c|}{ Abnormal results } \\
\hline \multirow{2}{*}{ Serum albumin } & 5.4 & $3.5-5.0 \mathrm{~g} / \mathrm{l}$ & Jun 2020 \\
\hline & 4.5 & $3.8-4.8 \mathrm{~g} / \mathrm{l}$ & Aug 2020 \\
\hline \multicolumn{4}{|l|}{ Antiphospholipid antibodies } \\
\hline \multirow{3}{*}{ Anti-beta-2 glycoprotein-1 IgM } & 36 & \multirow{3}{*}{ 0-32 GPI IgM } & Aug 2020 \\
\hline & 47 & & Dec 2020 \\
\hline & 59 & & Mar 2021 \\
\hline \multirow{3}{*}{$\begin{array}{l}\text { Anti-phosphatidylethanolamine } \\
\text { IgM }\end{array}$} & 56.5 & \multirow{3}{*}{$<12 \mathrm{U} / \mathrm{ml}$} & Aug 2020 \\
\hline & 22.6 & & Dec 2020 \\
\hline & 57.4 & & Mar 2021 \\
\hline \multirow{3}{*}{ Anti-prothrombin IgG } & 27 & \multirow{3}{*}{ 0-20 G units } & Aug 2020 \\
\hline & 5 & & Dec 2020 \\
\hline & NP & & Mar 2021 \\
\hline \multirow{3}{*}{ Anti-phosphatidylserine lgG } & 15 & \multirow{3}{*}{$0-11$ GPS IgG } & Aug 2020 \\
\hline & 11 & & Dec 2020 \\
\hline & 13 & & Mar 2021 \\
\hline \multicolumn{4}{|l|}{ Mast cell mediators } \\
\hline Serum prostaglandin $\mathrm{D} 2$ & 181 & $35-115 \mathrm{pg} / \mathrm{ml}$ & Aug 2020 \\
\hline \multirow{3}{*}{ Chromogranin A } & 205.3 & \multirow{3}{*}{$0-101.8 \mathrm{ng} / \mathrm{ml}$} & Aug 2020 \\
\hline & 255.1 & & Dec 2020 \\
\hline & 282.3 & & Mar 2021 \\
\hline
\end{tabular}

Table 1. Patient test results

NP: not performed. Normal test results included: transthoracic echocardiogram with bubble study, pulmonary function testing, chest CT scan, complete blood count, electrolytes, creatinine, liver function tests, random cortisol, thyroid function tests, glycosylated haemoglobin, creatine phosphokinase, aldolase, heavy metals (blood lead, arsenic, mercury), iron studies, erythrocyte sedimentation rate, C-reactive protein, urinalysis, HIV, chronic hepatitis panel, Lyme antibody panel, RPR, vitamins B1, B6, B12 and D, serum immunoglobulins and protein electrophoresis, antinuclear antibodies (ANA/ENA), complements C3, C4, rheumatoid factor/anti-CCP, novel Sjogren's panel (except for a single transient slightly positive antibody), thyroid antibodies, coeliac antibodies, paraneoplastic antibody panel, anti-lgE receptor antibody, lupus anticoagulant, anti-cardiolipin lgG/M/A, anti-beta-2 glycoprotein-1 lgG/A, antiphosphatidylethanolamine lgG/A, anti-phosphatidylserine lgM/lgA, anti-phosphatidylserine-prothrombin lgG/M, tryptase, plasma histamine and lgE food and environmental panels. 
The patient was evaluated by the author in August 2020. Her history suggested dysautonomia and MCAS. Stand testing was performed and she met the diagnostic criteria for POTS ${ }^{[6]}$ with an increase in heart rate from supine to standing of 55 bpm. Given the disabling nature of her illness, she underwent laboratory testing for underlying causes of her POTS (Table 1). There were four positive aPL, including 3 that have remained positive for more than a year after illness onset. There was also positive testing for two different mast cell mediators-serum prostaglandin D2 and chromogranin A.

Treatment for POTS, MCAS and aPL-positivity has resulted in significant improvement, despite the patient continuing to test positive for POTS by stand testing in January 2021, when her POTS treatment were held. She is now able to work 12-hour days functioning at 85-90\% of her prior baseline, and with MCAS abortive therapy, she functions at 70\% of normal during flares compared to previously being bed-bound. Her current medical regimen includes aspirin $162.5 \mathrm{mg}$ daily (improves cognitive dysfunction), sodium 4000 mg daily, amphetamine salts 5 mg daily for POTS, cetirizine 20 mg BID (more to abort flares), famotidine 40 mg BID, quercetin 1500 mg TID, vitamin C 500 mg TID, vitamin D3 6000 units daily, nasal cromolyn BID, diamine oxidase two tablets TID, diphenhydramine as needed for flares, and a low histamine, anti-inflammatory diet. She has also started hydroxychloroquine $200 \mathrm{mg}$ daily due to multiple persistent aPL, which might provide further symptomatic improvement.

\section{DISCUSSION}

This patient's history shows that post-COVID POTS may be very prolonged and possibly chronic. This is the first report describing the persistence of of multiple aPL more than one year after COVID-19 in a patient with clinical manifestations that have been published in association with aPL, that is, POTS. Autonomic neuropathy may be the initial manifestation of aPL-positivity ${ }^{[7]}$. Skin biopsy evaluating for suspected autonomic neuropathy was not performed because it would not change management. An abnormal skin biopsy is helpful for insurance approval when considering a trial of intravenous immunoglobulin, but as the present case illustrates, not all patients with autoimmunity and dysautonomia require immunoglobulin therapy.

APL-positivity in acute COVID-19 has been published by several groups ${ }^{[8,9]}$. Infections are known to induce transient aPL production--a phenomenon which led to the requirement for persistent aPL positivity for $\geq 12$ weeks before considering a diagnosis of antiphospholipid syndrome (APS) ${ }^{[10]}$. Only two studies have reported repeat aPL testing in acute COVID-19 patients. In one, 9 of 10 patients were aPLnegative 1 month later ${ }^{[8]}$ and in another ${ }^{[9]}$, three patients had persistent aPL-positivity, but only as late as 77 days after illness onset.

The persistent presence of aPL has important clinical implications, including an increased thrombotic risk as well as risk of pregnancy morbidity. While thrombosis and/or specific pregnancy morbidity are required for a 'definite' diagnosis of APS ${ }^{[10]}$, the identification of aPL provides the opportunity to prevent thrombosis by avoiding exogenous oestrogen, controlling for vascular risk factors which act synergistically with aPL, and the use of thromboprophylaxis postoperatively or postpartum. In women of childbearing age, aPL identification provides the opportunity to reduce pregnancy morbidity.

This patient also met Global Consensus-2 criteria ${ }^{[11]}$ for MCAS. Afrin et al. ${ }^{[4]}$ recently published their hypothesis that excessive MCA in people with unrecognized and thus untreated MCAS may be at the root of severe acute as well as long-haul COVID-19. This patient fits their hypothesis. Medications which inhibit MCA have demonstrated efficacy and/or are being studied in COVID-19 [4].

The specific diagnoses of POTS, MCAS and aPL-positivity were critical to achieving clinical improvement in this case and suggest that patients with prolonged symptoms after COVID-19 should undergo stand testing to evaluate for POTS and serological testing for autoimmunity. Owing to the limitations of MCAS testing as well as the excellent safety profile of most agents used in the treatment of MCAS ${ }^{[11]}$, consideration should be given to empiric treatment trials in long-haul COVID-19 patients with multisystem allergic and/or inflammatory symptoms suggesting MCAS. Patients with persistent aPL-positivity and migraine may respond dramatically to antithrombotic therapy ${ }^{[12]}$. Lastly, definite autoimmunity in a patient with severely disabling dysautonomia not improving with conservative therapies would support a trial of intravenous immunoglobulin which often results in dramatic improvement in this context ${ }^{[13]}$.

\section{CONCLUSION}

While SARS-CoV-2, like other autoimmune triggers, may incite a pathological innate and/or acquired immune response that may be prolonged, proper diagnoses and directed management may result in clinical improvement. Large studies of long-haul COVID-19 patients are imperative to better understand these complex multisystem disorders. 